
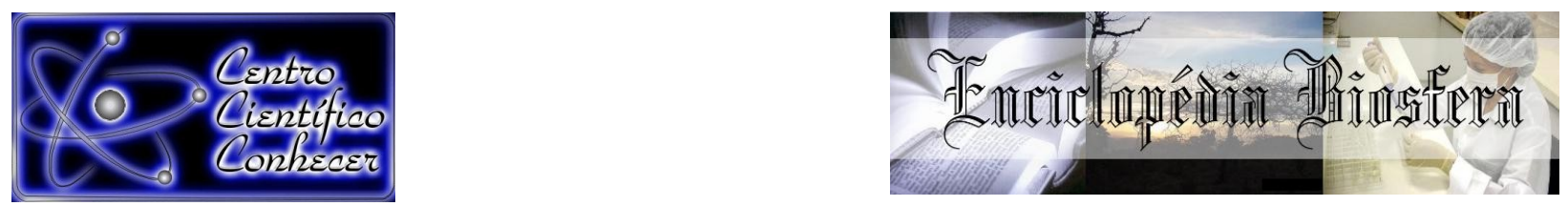

\title{
ESTUDO CIENCIOMÉTRICO SOBRE PREVENÇÃO QUATERNÁRIA
}

\author{
Lívia Karling Moreschi ${ }^{1}$, Mariane Hernandes Scandalo², Marcelo Picinin Bernuci ${ }^{3}$, \\ Mirian Ueda Yamaguchi ${ }^{4}$ \\ 1 Graduanda do Curso de Medicina do Centro Universitário de Maringá \\ (liviakm23@gmail.com). Paraná-Brasil \\ 2 Graduanda do Curso de Medicina do Centro Universitário de Maringá
}

3 Prof. Doutor do Programa de Pós-Graduação em Promoção da Saúde do Centro Universitário de Maringá e Pesquisador do Instituto Cesumar de Ciência, Tecnologia e Inovação

4 Prof. Doutora do Programa de Pós-Graduação em Promoção da Saúde do Centro Universitário de Maringá e Pesquisadora do Instituto Cesumar de Ciência, Tecnologia e Inovação

\section{Recebido em: 06/04/2018 - Aprovado em: 10/06/2018 - Publicado em: 20/06/2018 DOI: 10.18677/EnciBio_2018A99}

\begin{abstract}
RESUMO
Prevenção quaternária é prevenir o excesso do cuidado em saúde e evitar intervenções desnecessárias, reduzindo danos, por meio de técnicas e práticas clínicas qualificadas e personalizadas. O objetivo desta pesquisa foi verificar a produção científica nacional e internacional sobre o tema prevenção quaternária. Realizou-se um estudo cienciométrico nas bases de dados Scielo, Pubmed e ScienceDirect utilizando o termo "prevenção quaternária" no mês de fevereiro de 2018. Após análise, foram identificados 34 artigos correspondentes ao tema. 0 principal eixo temático foi o de conceito, com explicações sobre o termo e suas aplicações. O número de publicações vem crescendo, com destaque no ano de 2014. O país com o maior número de artigos foi a Espanha que totalizou $29,4 \%$ das publicações. O estudo identificou uma baixa produção de artigos sobre a temática ao longo dos anos, algo preocupante, visto que a prevenção quaternária se torna cada vez mais necessária na pratica médica.
\end{abstract}

PALAVRAS-CHAVE: iatrogenia, prevenção quaternária, promoção da saúde.

\section{SCIENTOMETRIC STUDY ON QUATERNARY PREVENTION}

\begin{abstract}
Quaternary prevention is to prevent excess health care and avoid unnecessary interventions, reducing harm, through qualified and personalized care techniques and practices. The objective of this research was to verify the national and international scientific production on the subject of quaternary prevention. A scientiometric study was carried out in the databases Scielo, Pubmed and ScienceDirect using the term "quaternary prevention" in the month of February 2018. After analysis, 34 articles corresponding to the theme were identified. The main thematic axis was the concept, with explanations about the term and its applications. The number of publications is growing, especially in 2014. The country with the largest number of articles was Spain, which accounted for $29.4 \%$ of publications. The study identified a low
\end{abstract}


production of articles on the theme over the years, a matter of concern, as quaternary prevention becomes increasingly necessary in medical practice.

KEYWORDS: iatrogenic, quaternary prevention, health promotion.

\section{INTRODUÇÃO}

Prevenção quaternária (P4), refere-se ao manejo do excesso de intervenção e medicalização, tanto diagnóstica quanto terapêutica, o qual foi inicialmente proposto pelo médico belga Marc Jamoulle em 1990, (JAMOULLE; ROLAND, 1995) e definido em 2003, pela Organização Mundial de Colégios Nacionais, Academias e Associações Acadêmicas de Médicos gerais/Médicos de Família (WONCA) como um conceito que visa proteger os pacientes da intervenção médica desnecessária e prevenir iatrogenias (BENTZEN, 2003).

Em 2014, a Organização Mundial da Saúde, considera que as ações de identificação de pacientes em risco de sobremedicalização fazem parte do contexto da P4, e pode, portanto, proteger estes pacientes de mais intervenções em saúde (CHINITZ; RODWIN, 2014). Atualmente, após inúmeros debates sobre as repercussões sociais e econômicas da aplicação da P4 no cotidiano da atuação médica, Jamoulle considera que o termo pode ser aplicado como a prevenção da própria medicina, cujo entendimento poderia ajudar a controlar custos econômicos e humanos dos cuidados de saúde (JAMOULLE, 2015). Finalmente, assume-se que a P4 vai além de prevenir sobrediagnóstico ou prevenção de sobretratamento, inclui prevenção de todos os tipos de danos associados às intervenções (MARTINS et al., 2018).

No Brasil, a necessidade da adoção e discussão do conceito da P4 no SUS emergiu especialmente no âmbito da atenção primária à saúde em decorrência das evidências de excesso de rastreamento, de solicitação de exames complementares e de medicalização de fatores de risco (NORMAN; TESSER, 2009). Ademais, as implicações sociais e econômicas das ações da P4 na atenção à saúde no país têm assumido constante debate, marcado por um manifesto em prol de uma Medicina isenta de conflitos de interesses por médicos de família e comunidade no I Seminário Brasileiro de Prevenção Quaternária (SILVA et al., 2014). A ideia de que o excesso de cuidados transforma pessoas saudáveis em "doentes" tem cada vez mais trazido à tona a relevância das implicações das ações de P4 no cenário da prática médica contemporânea brasileira e mundial (JAMOULLE, 2015; TESSER; NORMAN, 2016; TESSER, 2017).

Neste tocante, conceitos como "disease mongering, selling sickness e doctor shopping" que retratavam os lucros obtidos com pessoas doentes e não doentes, tem assumido maior espaço nas discussões acerca do tema da P4 (SANTOS, 2014). Isso se deve ao fato de que a execução da prática da P4 leva aos profissionais de saúde e aos pacientes o conhecimento sobre os possíveis danos sofridos por intervenções médicas (TOSCAS; TOSCAS, 2015). Dessa forma, reconhecer o estado da arte das pesquisas científicas que se direcionam para o entendimento das ações de P4 e suas implicações no contexto das práticas médicas contemporâneas pode favorecer a compreensão das repercussões dessa nova temática no contexto da saúde coletiva. Assim, o presente estudo direciona-se a compreender a dinâmica das publicações científicas, nacionais e internacionais, acerca da P4, focando na análise temporal e regional das publicações e nos temas e doenças abordados.

\section{MATERIAIS E MÉTODOS}


Trata-se de um estudo cienciométrico da literatura científica nacional e internacional sobre a Prevenção Quaternária. A pesquisa foi realizada nas bases de dados: a) United States National Library of Medicine (PubMed), acessada pelo site http://www.ncbi.nlm.nhi.gov/pubmed; b) Scientific Eletronic Library Online (SciELO), disponível na Biblioteca Virtual em Saúde (BVS) pelo site http://www.bireme.br; e c) ScienceDirect, acessada pelo site https://www.sciencedirect.com.

A procura pelos artigos nas bases de dados foi realizada durante o mês de fevereiro de 2018 utilizando o descritor em português "prevenção quaternária" e seu correspondente em inglês "quaternary prevention". Na base de dados ScienceDirect foi utilizado o filtro de dados Review articles e Research articles. Os artigos identificados foram selecionados e classificados por meio da análise dos títulos e resumos.

Foram excluídos os artigos com tema diferente do pesquisado, as cartas ao editor e os comentários sobre outras publicações. Após a leitura dos artigos, os dados cienciométricos foram classificados pelo ano de publicação, local de origem das publicações, eixos temáticos e doenças específicas que foram focadas nos estudos. Esses dados foram tabulados e organizados em planilhas do programa Microsoft Excel 2010 e apresentados de forma descritiva a partir da construção de fluxograma e gráficos de barras.

\section{RESULTADOS - Identificação e inclusão dos estudos}

Um total de 101 estudos foram identificados na pesquisa bibliográfica. Destes, 56 na base de dados PubMed, 18 na base de dados Scielo e 27 na base de dados SciencieDirect (Figura 01). Após exclusão de artigos duplicados e aplicação dos critérios de inclusão, foram selecionados 34 artigos.

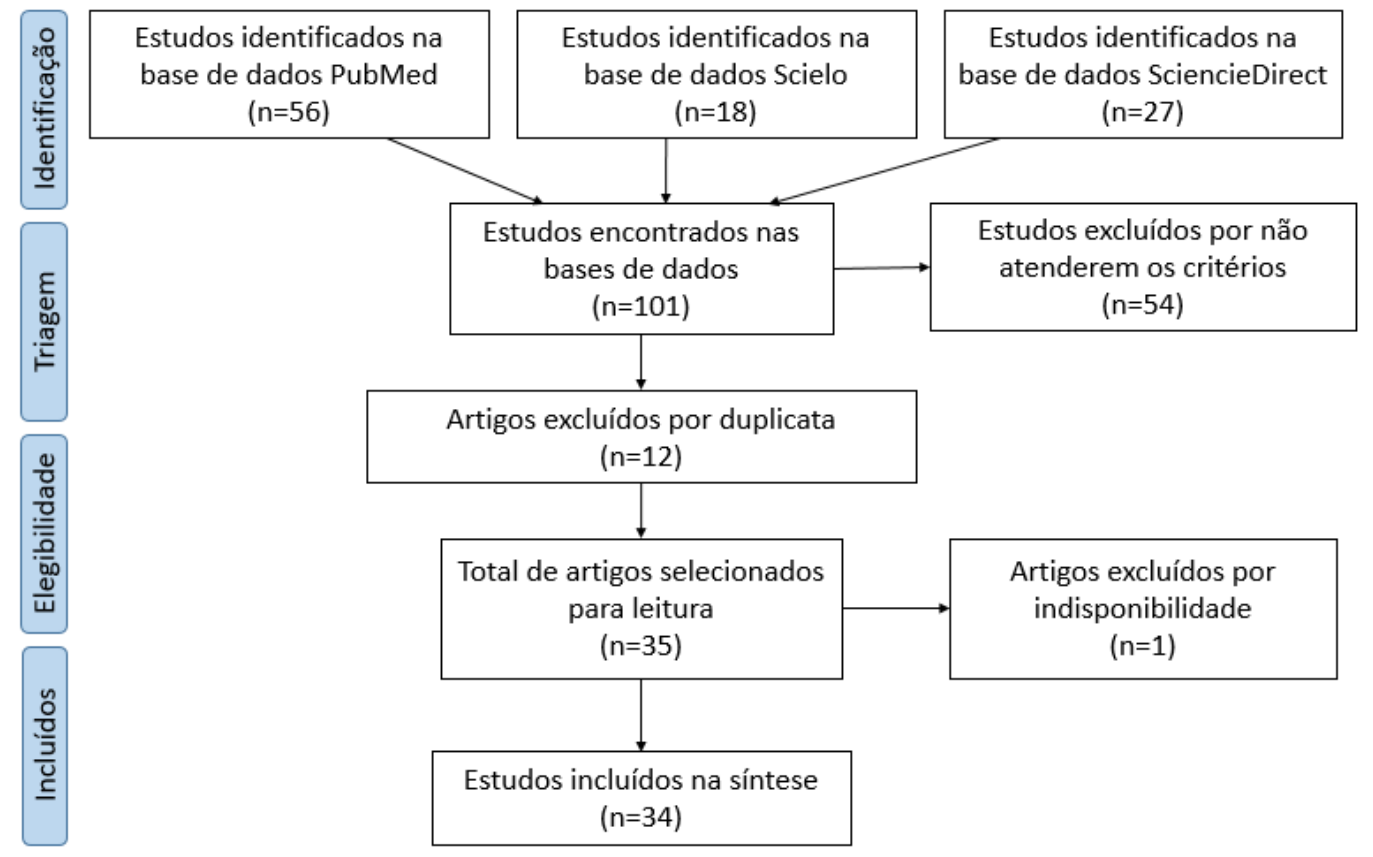

FIGURA 1. Fluxograma do processo de identificação e inclusão dos estudos.

\section{Análise temporal e regional das publicações}

Os dados referentes a análise temporal das publicações científicas estão organizados na Figura 02. Nota-se que, excluindo-se as publicações clássicas de definição do termo, os estudos direcionados a aplicação da P4 se iniciaram a partir do ano de 2001, persistindo sem grande expressão numérica até 2012. Por outro ENCICLOPÉDIA BIOSFERA, Centro Científico Conhecer - Goiânia, v.15 n.27; p.114 
lado, nota-se um grande incremento no número de publicações em 2014, o que se sustentou pelos anos seguintes. A redução do volume de publicações no ano de 2018 se deve ao fato de que apenas os meses de janeiro e fevereiro do referido ano foram contabilizados no processo de busca bibliográfica.

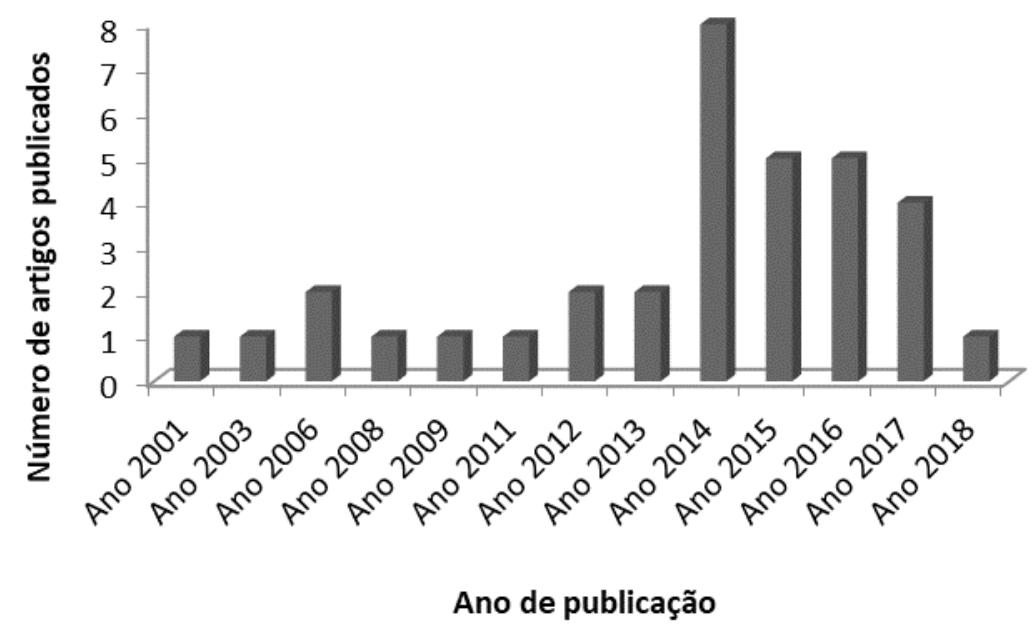

FIGURA 2. Perfil temporal das publicações científicas. Número de artigos publicados anualmente no período de 2001 a 2018 sobre a Prevenção Quaternária.

Os dados referentes a análise regional das publicações científicas (local de origem dos estudos) estão organizados na Figura 03. Nota-se que no período investigado, os estudos originados da Espanha foram os que mais contribuíram para o montante de artigos publicados sobre a P4 (29,4\%), seguido da Índia $(14,7 \%)$ e Brasil (14,7\%). Outros países que também publicaram sobre o tema foram: Portugal, Peru, Argentina, Alemanha, Coreia, Croácia, Estados Unidos, Franca, Itália e Noruega, porém como menor expressividade.

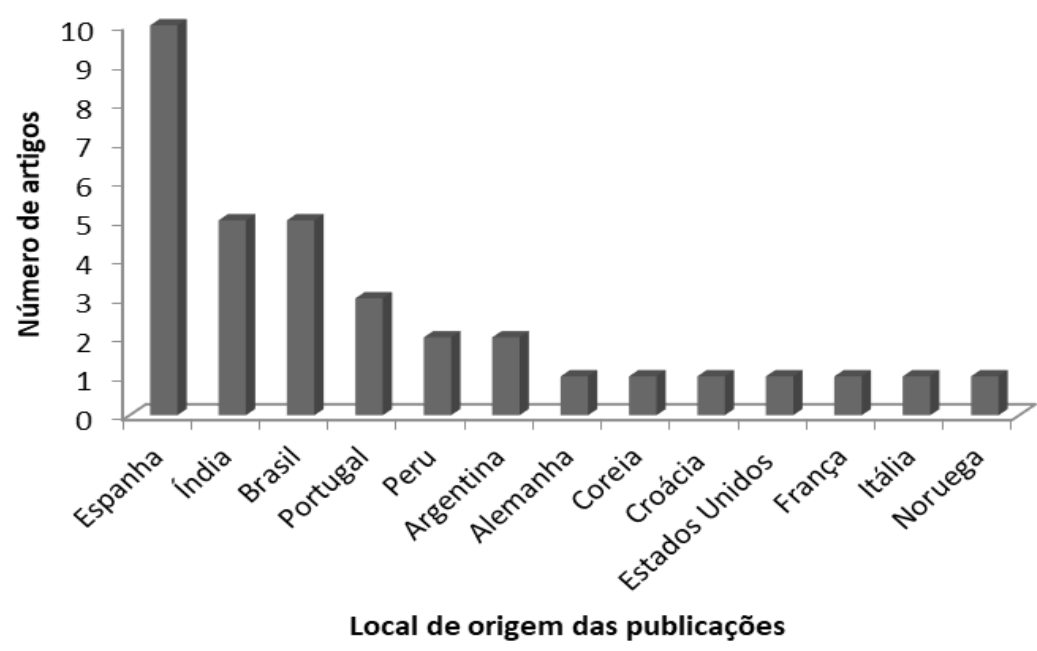

FIGURA 3. Perfil regional das publicações científicas. Número de artigos publicados por pesquisadores de diferentes países no período de 2001 a 2018 sobre a Prevenção Quaternária.

\section{Análise temática das publicações}


Os dados referentes ao perfil temático abordado nas publicações científicas estão representados na Figura 04. Nota-se uma prevalência de artigos que exploram o conceito de prevenção quaternária, seguido por trabalhos que relacionam a prevenção quaternária com uma determinada doença. Na sequência, observa-se um grande volume de estudos que se destinaram a discutir os aspectos éticos da P4. Temas importantes como iatrogenia e medicalização foram pouco abordados nos estudos analisados.

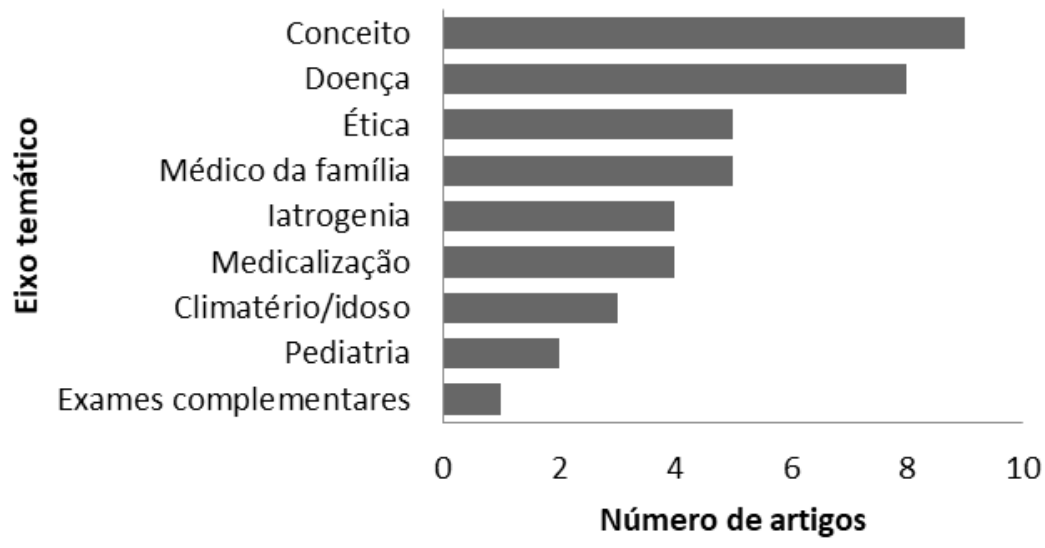

FIGURA 4. Perfil temático abordado nas publicações científicas. Número de artigos publicados por eixo temático no período de 2001 a 2018 sobre a Prevenção Quaternária.

\section{DISCUSSÃO}

Diante do fato de que a P4 consiste na identificação de pessoas em risco de excessiva medicalização e sua proteção contra novas intervenções desnecessárias, evitando danos iatrogênicos, a análise do estado da arte das produções científicas sobre este tema torna-se relevante no contexto atual de enfrentamento dos problemas de saúde sob constantes restrições orçamentárias. Dessa forma, foi constatado na presente análise cienciométrica, uma certa carência de publicações científicas referentes às implicações da P4 no contexto da saúde. Além disso, embora os países da Europa concentrem grande parte das publicações referentes ao tema, o Brasil tem se despontado como um embaixador do tema na América Latina, visto que tem contribuído com número significativo de publicações relacionadas com a $\mathrm{P} 4$.

Pelo fato de ser um termo relativamente novo, nota-se também uma prevalência de trabalhos que explanam sobre o conceito, explicando o que é e como a P4 pode ser praticada nas diferentes atribuições da medicina. De fato, foi identificado na presente análise cienciométrica que o início expressivo das publicações se deu no ano de 2001, período subsequente à publicação oficial do termo pelo médico Jamoulle em 1990 (JAMOULLE \& ROLAND, 1995). No entanto, o maior volume de publicações é apenas notado após o ano de 2014, presumivelmente em resposta à disseminação feita pelo Comitê Internacional de Classificação da WONCA (WICCA) através de dois grandes eventos, a Conferência WONCA Europe Basel em 2010 e Conferência Mundial WONCA em Praga em 2013, que contaram com oficinas e palestras sobre P4 em diversos idiomas (JAMOULLE, 2015).

Neste interim, a Espanha teve um destaque no número de publicações, que pode ter uma relação com a importância dada à atenção primária neste país desde o 
plano estratégico formulado em 2000, mesma época da proposta da P4, ocasionando uma reestruturação da saúde já com enfoque na diminuição de excessos médicos (CAMPOS et al., 2016). Além disso, os gastos públicos com prescrições farmacêuticas somaram $20 \%$ do total gasto com a saúde deste país, o que deve ter gerado preocupação tanto financeira quanto médica, visto que muitas vezes, esses medicamentos não são necessários para uma melhor qualidade de vida a longo prazo (CARMONA et al., 2015) e pode, portanto, ter promovido maior repercussão do tema no âmbito da comunidade científica.

No Brasil, este cenário também repercutiu de maneira semelhante. Os dados de excesso de solicitação de exames complementares e de indicação medicamentosa para indivíduos sob fatores de riscos de doenças levaram para a atenção primária à saúde o tema da P4 (NORMAN; TESSER, 2009). Os movimentos de médicos e profissionais ligados a medicina da família e da comunidade propulsionaram debates sobre as questões éticas referentes à prática médica contemporânea, dando relevância da aplicabilidade dos conceitos da P4 para o âmbito social e econômico (SILVA et al., 2014). Por conseguinte, nota-se uma motivação da inserção da comunidade científica nacional no aprofundamento técnico-científico do tema, o que pode ser visto na análise do perfil regional das publicações no presente estudo cienciométrico.

De fato, quando se aprofunda na análise dos temas abordados pelos estudos científicos, questões relacionadas a ética assumem grande destaque. Como o princípio fundamental da medicina é primum non nocere (primeiro não fazer mal), é evidente a dificuldade em se dissociar a P4 do tema ética. Levando em consideração a evolução do processo de atenção em saúde, com a massiva tecnização da atividade, é de fundamental importância a discussão ética dentro de uma prevenção que visa a diminuição dos malefícios das intervenções médicas. Uma outra visão ética da prevenção quaternária, recai sobre outro eixo temático encontrado nas publicações, o excesso de medicalização, visto que muitas vezes a relação entre médicos e a indústria farmacêutica possui muitos interesses secundários. De acordo com Moynihan (2003), 80 a 95\% dos médicos conversam com representantes farmacêuticos regularmente mesmo sabendo que os dados fornecidos por eles são exageradamente positivos. Além disso, algumas indústrias farmacêuticas utilizam vários artifícios para incentivar os médicos a prescreverem determinado medicamento, como a oferta de viagens, equipamentos, jantares e outros benefícios (MASSUD, 2010). Essas constatações reafirmam a necessidade emergente de se discutir e implementar as ações de P4 na prática da medicina contemporânea.

Hodiernamente, o tema "iatrogenia" teve menos destaque nos trabalhos publicados, apesar de ser uma complicação custosa para o sistema de saúde, como apontado por Porto et al. (2010) onde $64,1 \%$ dos eventos adversos ocorridos nos hospitais do Brasil foram considerados evitáveis e geraram um valor médio de custo por paciente de 19,5\% superior ao paciente sem evento adverso. Além disso, a iatrogenia pode ser custosa para o paciente: entre 104.187 a 434.112 possíveis óbitos estão associados a eventos adversos hospitalares/ano no Brasil (COUTO et al., 2016). Ainda em relação aos custos que a P4 pode diminuir, Pires (2011) afirma que um cuidado centrado no paciente diminui os gastos anuais da saúde por paciente de $\$ 1435.00$ para $\$ 948.00$ por reduzir a utilização dos serviços de saúde (hospitalizações, consultas hospitalares, exames complementares de diagnóstico e utilização de especialidades hospitalares). O fato de que os estudos científicos têm pouco abordado a relação entre $\mathrm{P} 4$ e iatrogenia reacende a necessidade da 
constante discussão da implementação das ações de P4 tanto no campo da prática médica como da científica.

Quanto a relação da P4 com determinadas doenças, o câncer teve maior destaque, provavelmente pela recente revisão de protocolos de rastreio com estudos que mostraram uma mudança muito pequena na mortalidade especifica de determinados cânceres que não compensava os riscos relacionados à exames invasivos para confirmação do diagnóstico, às sequelas do tratamento e o impacto psicológico de um resultado falso-positivo (MODESTO et al., 2017). Da mesma forma, o destaque dado à relação entre P4 e a saúde mental, chama a atenção quanto ao excesso de diagnósticos. Segundo Lobo e Rojo (2011), é preciso muita atenção para não diagnosticar como transtornos mentais um paciente que tem apenas sofrimentos vinculados ao dia-a-dia, bem como muito cuidado com os tratamentos e diagnósticos excessivos para não estigmatizar o paciente, dificultando sua vida em sociedade. Neste sentido, fica evidente, a ideia de que o excesso de cuidados transforma pessoas saudáveis em doentes já observado em pesquisas clássicas de revisão do termo P4 (JAMOULLE, 2015; TESSER; NORMAN, 2016; TESSER, 2017).

De forma geral, a análise cienciométrica aqui apresentada demonstra o estado da arte das publicações científicas sobre o tema P4 e chama a atenção quanto ao pequeno volume de artigos publicados sobre o tema, mesmo em um período em que avanços constantes têm acometido a área médica e farmacêutica, que induzem os profissionais a utilizar as novidades com a promessa de serem o melhor para o paciente. As discussões acerca da P4 evidenciam que muitas vezes, esses avanços podem trazer mais danos do que benefícios, destacando, portanto, a necessidade imediata de maiores discussões sobre as ações de P4 na prática da medicina atual.

\section{REFERÊNCIAS}

BENTZEN, N. WONCA dictionary of general/family practice. Copenhagen: Maanedskift Lager; 2003.

CAMPOS, J. J. B.; FORSTER, A. C.; FREIRE FILHO, J. R. Lições aprendidas na comparação dos sistemas de saúde brasileiro e espanhol. Revista de Saúde Pública do Paraná, v. 17, n. 1, p. 121-129, 2016. Disponível em: < http://dx.doi.org/10.22421/1517-7130.2016v17n1p121>. DOI: 10.5433/15177130.2016v17n1p121.

CARMONA, J. H.; CRUZ I. A.; PARRILLA, F. R. Un modelo de deprescripción prudente. Medicina Clínica (Barcelona), v. 144, n. 8, p. 362-369, 2015. Disponível em:

<https://doi.org/10.1016/j.medcle.2015.11.013>.

Doi:10.1016/j.medcli.2014.02.026.

CHINITZ, D. P.; RODWIN, V. G. On Health Policy and Management (HPAM): mind the theory-policy-practice gap. International Journal of Health Policy and Management, 2014; $\quad$ v. 3, n. 7, p. 361 - 363. Disponível em: <http://ijhpm.com/article_2919_39cc81079e4f6a09446da27799778bd2.pdf> DOI: 10.15171/ijhpm.2014.122

COUTO R. C.; PEDROSA, T. M. G.; ROSA, M. B. Erros acontecem: a força da transparência no enfrentamento dos eventos adversos assistenciais em pacientes 
hospitalizados. Instituto de Estudos de Saúde Suplementar. Belo Horizonte, 2016. Disponível em: http://documents.scribd.com.s3.amazonaws.com/docs/5x5i1j985c5jwcsp.pdf.

JAMOULLE, M. Prevenção quaternária: primeiro não causar dano. Revista Brasileira de Medicina de Família e Comunidade, v. 10, n. 35, p. 1-3, 2015. Disponível em: <https://orbi.uliege.be/bitstream/2268/176760/1/1064-7076-1-PB.pdf> DOI: https://doi.org/10.5712/rbmfc10(35)1064

JAMOULLE, M.; ROLAND, M. Quaternary prevention. WICC annual workshop: Hongkong, Wonca congress proceedings, 1995. Disponível em: http://hdl.handle.net/2268/173994.

JAMOULLE, M. Quaternary prevention, an answer of family doctors to overmedicalization. International Journal of Health Policy and Management, v. 4, n. 2, p. 61-64, 2015. Disponível em: $<$ https://www.ncbi.nlm.nih.gov/pmc/articles/PMC4322627/> DOI:10.15171/ijhpm.2015.24.

LOBO, A. O.; ROJO, V. I. latrogenia y prevención cuaternaria en salud mental. Revista Española de Salud Pública, Madrid, v. 85, n. 6, p. 513-525, dic. 2011 Disponível em: <http://scielo.isciii.es/scielo.php? script=sci_arttext\&pid=S1135-57272011000600002\&lng=es\&nrm=iso>.

MARTINS, C.; GODYCKI-CWIRKO, M.; HELENO, B.; BRODERSEN, J. Quaternary prevention: reviewing the concept. European Journal of General Practice, v. 24, n. 1, p. 106-111, 2018. Disponível em: < https://www.ncbi.nlm.nih.gov/pubmed/29384397> DOI: 10.1080/13814788.2017.142 2177

MASSUD, M. Conflito de interesses entre os médicos e a indústria farmacêutica. Revista Bioética, v. 18, n.1, p. 75 - 91, 2010. Disponível em: < http://revistabioetica.cfm.org.br/index.php/revista_bioetica/article/view/537>.

MODESTO, A. A. D.; LIMA, R. L. B.; D'ANGELIS A. C.; AUGUSTO D. K. Um novembro não tão azul: debatendo rastreamento de câncer de próstata e saúde do homem. Interface (Botucatu) Botucatu, v. 22, n. 64, p. 251-262, 2018. Disponível

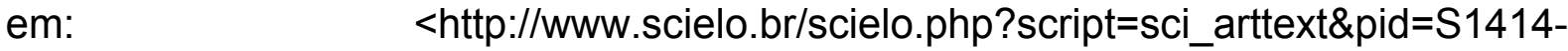
32832018000100251\&lng=en\&nrm=iso>. DOI: http://dx.doi.org/10.1590/180757622016.0288 .

MOYNIHAN, R. Who pays for the pizza? Redefining the relationships between doctors and drug companies. 1: Entanglement. British Medical Journal, v. 326, p. 1189 - 1192, 2003. Disponível em: < https://www.bmj.com/content/326/7400/1189>. DOI: https://doi.org/10.1136/bmj.326.7400.1189.

NORMAN, A. H.; TESSER, C. D. Prevenção quaternária na atenção primária à saúde: uma necessidade do Sistema Único de Saúde. Caderno de Saúde Pública, Rio de Janeiro, v. 25, n. 9, p. 2012-2020, 2009. Disponível em: $<$ http://www.scielo.br/scielo.php?script=sci_arttext\&pid=S0102- 
311X2009000900015\&lng=en\&nrm=iso>. DOI: http://dx.doi.org/10.1590/S0102$311 \times 2009000900015$.

PIRES, P. Medicina centrada no paciente: melhor qualidade com menores custos. Revista Portuguesa de Clínica Geral, Lisboa, v. 27, n. 5, p. 482-486, 2011. Disponível em <http://www.scielo.mec.pt/scielo.php?script=sci_arttext\&pid=S0870$71032011000500012 \&$ Ing=pt\&nrm=iso $>$.

PORTO, S.; MARTINS, M.; MENDES, W.; TRAVASSOS, C. A magnitude financeira dos eventos adversos em hospitais no Brasil. Revista Portuguesa de Saúde Pública, v. 10, p. $74 \quad-\quad 80, \quad 2010$. Disponível em: http://www.elsevier.pt/pt/revistas/revista-portuguesa-saude-publica-323/artigo/amagnitude-financeira-dos-eventos-adversos-em-hospitais-X0870902510898606.

SANTOS, J. A. A "cadeia alimentar" no mercado da saúde: do disease mongering ao doctor shopping. Revista Brasileira de Medicina de Família e Comunidade, Rio de Janeiro, v. 9, n. 31, p. 210-212, 2014. Disponível em: https://www.rbmfc.org.br/rbmfc/article/view/853.

https://doi.org/10.5712/rbmfc9(31)853.

SILVA, A. L.; MANGIN, D.; PIZZANELLI, M.; JAMOULLE, M.; WAGNER, H. L.; SILVA, D. H., et al. Manifesto de Curitiba: pela Prevenção Quaternária e por uma Medicina sem conflitos de interesse. Revista Brasileira de Medicina de Família e Comunidade, v. 9, n. 33, p. 371-374, 2014. Disponível em: < https://www.rbmfc.org.br/rbmfc/article/view/1006>. https://doi.org/10.5712/rbmfc9(33)1006.

TESSER, C. D.; NORMAN, A. H. Differentiating clinical care from disease prevention: a prerequisite for practicing quaternary prevention. Caderno de Saúde Pública, Rio de Janeiro, v. 32, n. 10, e00012316, Oct. 2016. Disponível em $<$ http://www.scielo.br/scielo.php?script=sci_arttext\&pid=S0102-

311X2016001003001\&lng=en\&nrm=iso>. $\quad$ DOI: http://dx.doi.org/10.1590/0102$311 \times 00012316$.

TESSER, C. D. Why is quaternary prevention important in prevention?. Revista de Saúde Pública, São Paulo, v. 51, p. 116, dec. 2017. ISSN 1518-8787. Disponível em: $\quad<h t t p s: / / w w w . r e v i s t a s . u s p . b r / r s p / a r t i c l e / v i e w / 141548>$. DOI: http://dx.doi.org/10.11606/S1518-8787.2017051000041.

TOSCAS, F. S; TOSCAS, F. Sobrediagnóstico e suas implicações na engenharia clínica. Revista Bioética, Brasília, v. 23, n. 3, p. 535-541, Dec. $2015 . \quad$ Disponível em: $\quad<h t t p: / / w w w . s c i e l o . b r / s c i e l o . p h p ? s c r i p t=s c i=a r t t e x t \& p i d=S 1983-$ 80422015000300535\&lng=en\&nrm=iso>. D Doi: http://dx.doi.org/10.1590/198380422015233090 . 